\title{
Supporting Information \\ Density, sound speed and viscosity of dihydropyridine derivatives in dimethyl sulfoxide at different temperatures
}

\author{
Shipra Baluja* and Rahul M. Talaviya \\ Physical Chemistry Laboratory, Department of Chemistry, \\ Saurashtra University, Rajkot-360005 (Gujarat), India.
}

*Corresponding author: Tel; No. +91-9687692918

E-mail: shipra_baluja@rediffmail.com 
Figure S $1{ }^{1} \mathrm{H}$ NMR spectrum of VMDP-1 along with peak integral
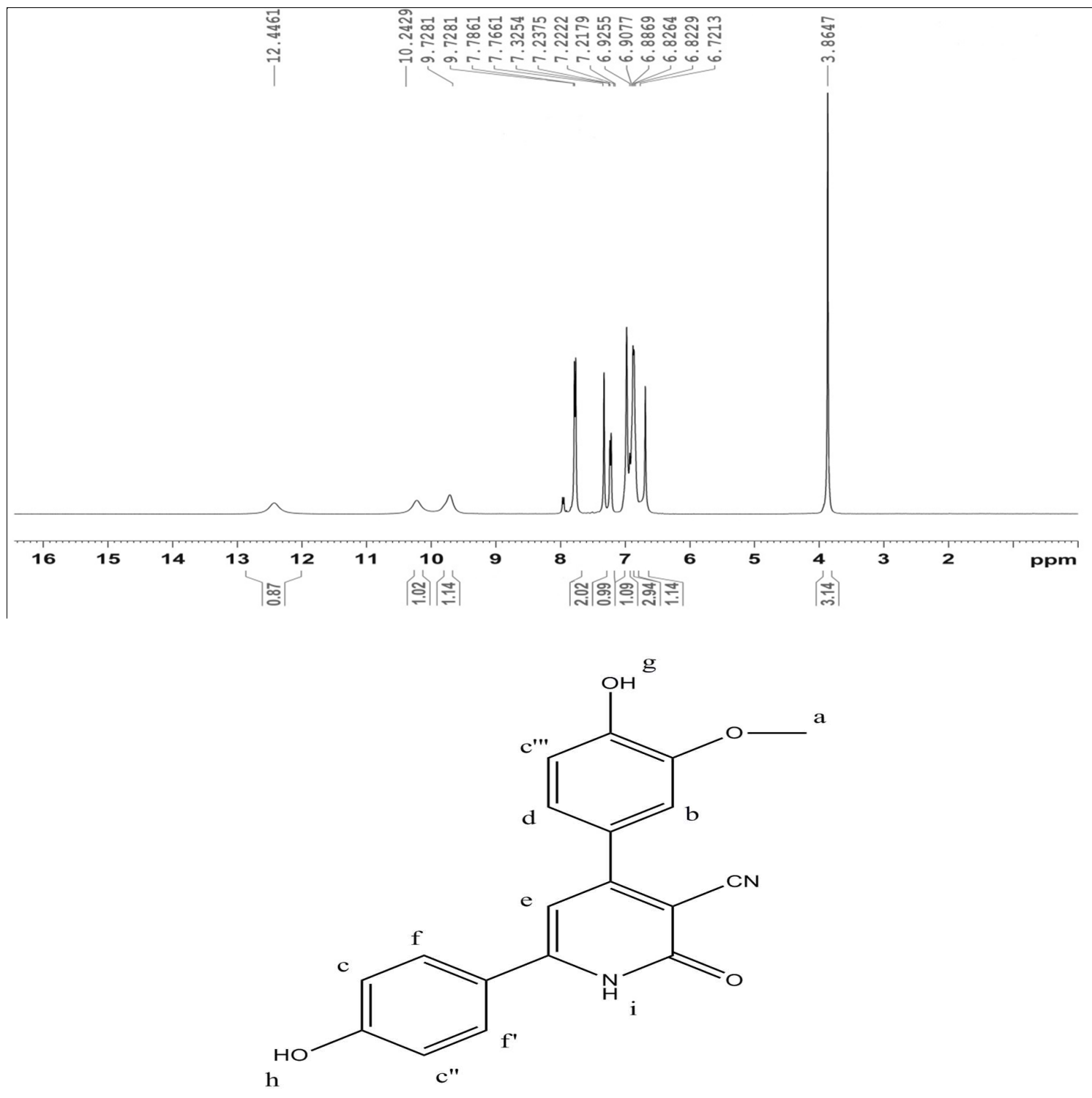

\begin{tabular}{|c|c|c|c|c|c|}
\hline $\begin{array}{c}\text { Signal } \\
\text { No. }\end{array}$ & $\begin{array}{c}\text { Signal position } \\
(\delta \mathrm{ppm})\end{array}$ & $\begin{array}{c}\text { Relative no. } \\
\text { of protons }\end{array}$ & Multiplicity & inference & $J(\mathrm{~Hz})$ \\
\hline 1 & 3.8647 & 3 & singlet & $-\mathrm{OCH}_{3}$ & - \\
\hline 2 & 6.7213 & 1 & singlet & Ar-Hb & - \\
\hline 3 & $6.8229-6.9255$ & 3 & multiplet & Ar-Hcc'c” & - \\
\hline 4 & $7.2179-7.2375$ & 1 & doublet & Ar-Hd & 7.84 \\
\hline 5 & 7.3254 & 1 & singlet & Ar-He & - \\
\hline 6 & $7.7661-7.7861$ & 2 & doublet & Ar-Hff & 8.24 \\
\hline 7 & 9.7281 & 1 & singlet & Ar-Hg & - \\
\hline 8 & 10.2429 & 1 & singlet & Ar-Hh & - \\
\hline 9 & 12.4461 & 1 & singlet & -NHi & - \\
\hline
\end{tabular}


Figure S2 ${ }^{1} \mathrm{H}$ NMR spectrum of VMDP-2 along with peak integral

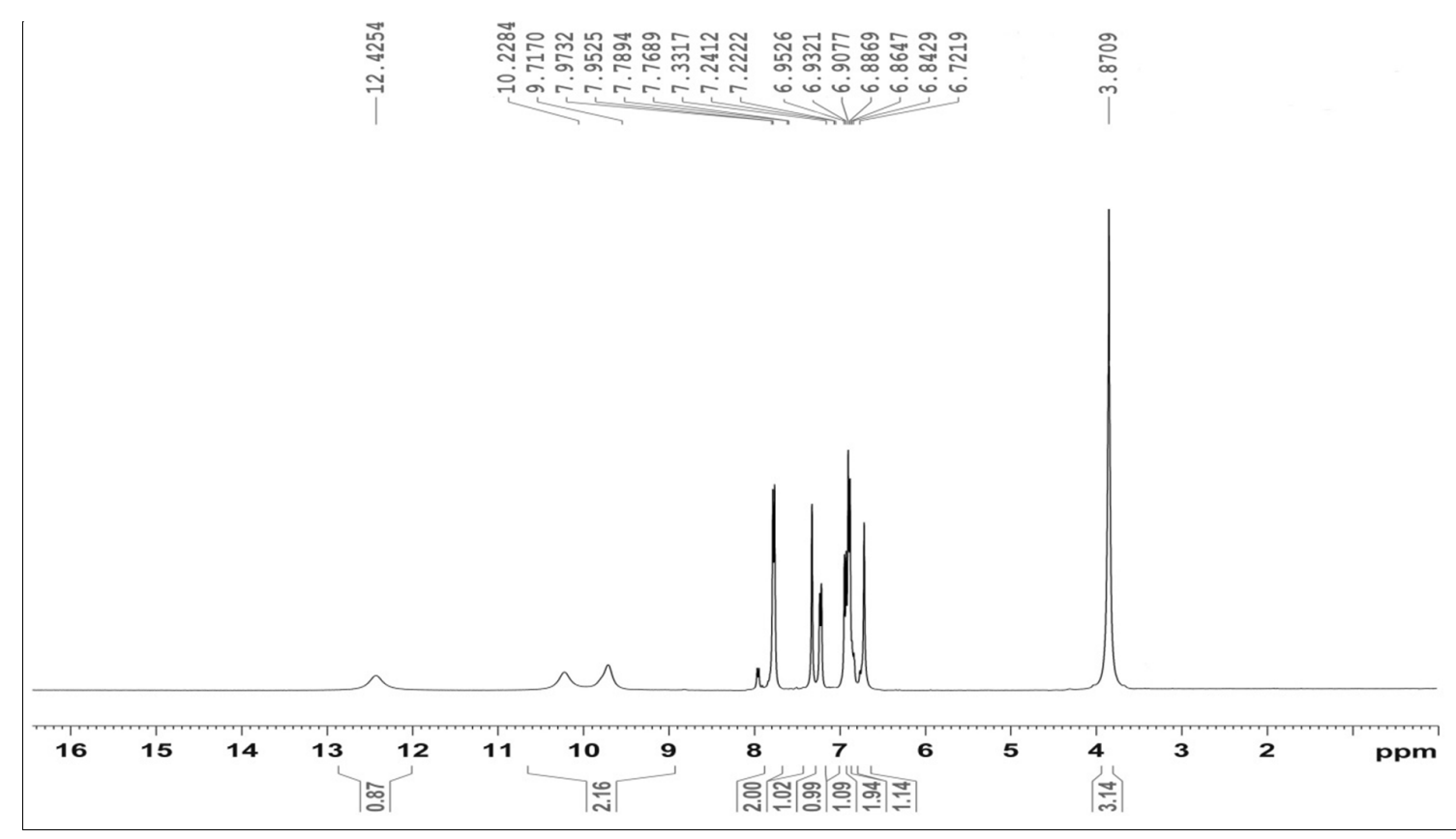<smiles>COc1cc(-c2cc(-c3ccccc3O)[nH]c(=O)c2C#N)ccc1O</smiles>

\begin{tabular}{|c|c|c|c|c|c|}
\hline $\begin{array}{c}\text { Signal } \\
\text { No. }\end{array}$ & $\begin{array}{c}\text { Signal position } \\
(\delta \mathrm{ppm})\end{array}$ & $\begin{array}{c}\text { Relative no. } \\
\text { of protons }\end{array}$ & Multiplicity & inference & $J(\mathrm{~Hz})$ \\
\hline 1 & 3.8709 & 3 & singlet & $-\mathrm{OCH}_{3}$ & - \\
\hline 2 & 6.7219 & 1 & singlet & Ar-Hb & - \\
\hline 3 & $6.8429-6.9526$ & 2 & multiplet & Ar-Hcc' & - \\
\hline 4 & $7.2222-7.2412$ & 1 & doublet & Ar-Hd & - \\
\hline 5 & 7.3317 & 1 & singlet & Ar-He & 7.6 \\
\hline 6 & $7.7689-7.7894$ & 1 & doublet & Ar-Hf & 8.6 \\
\hline 7 & $79525-7.9732$ & 2 & doublet & Ar-Hgg' & 8.2 \\
\hline 8 & 9.7170 & 1 & singlet & Ar-Hh & - \\
\hline 9 & 10.2284 & 1 & singlet & Ar-Hi & - \\
\hline 10 & 12.4254 & 1 & singlet & $-\mathrm{NHj}$ & - \\
\hline
\end{tabular}


Figure S3 ${ }^{1} \mathrm{H}$ NMR spectrum of VMDP-3 along with peak integral


\begin{tabular}{|c|c|c|c|c|c|}
\hline $\begin{array}{c}\text { Signal } \\
\text { No. }\end{array}$ & $\begin{array}{c}\text { Signal position } \\
(\delta \mathrm{ppm})\end{array}$ & $\begin{array}{c}\text { Relative no. } \\
\text { of protons }\end{array}$ & Multiplicity & inference & $J(\mathrm{~Hz})$ \\
\hline 1 & 3.8622 & 3 & singlet & $-\mathrm{OCH}_{3}$ & - \\
\hline 2 & 6.8806 & 1 & singlet & $\mathrm{Ar}-\mathrm{Hb}$ & - \\
\hline 3 & $6.9308-6.9514$ & 1 & doublet & Ar-Hc & 8.24 \\
\hline 4 & $7.2436-7.2684$ & 1 & doublet & Ar-Hd & 9.92 \\
\hline 5 & $7.3446-7.3487$ & 1 & singlet & Ar-He & - \\
\hline 6 & $7.6047-7.6259$ & 2 & doublet & Ar-Hff & 8.48 \\
\hline 7 & $7.9186-7.9389$ & 2 & doublet & Ar-Hgg' & 8.12 \\
\hline 8 & 9.7776 & 1 & singlet & Ar-Hi & - \\
\hline 9 & 12.7288 & 1 & singlet & -NHh & - \\
\hline
\end{tabular}


Figure S4 ${ }^{1} \mathrm{H}$ NMR spectrum of VMDP-4 along with peak integral


\begin{tabular}{|c|c|c|c|c|c|}
\hline $\begin{array}{c}\text { Signal } \\
\text { No. }\end{array}$ & $\begin{array}{c}\text { Signal position } \\
(\delta \mathrm{ppm})\end{array}$ & $\begin{array}{c}\text { Relative no. } \\
\text { of protons }\end{array}$ & Multiplicity & inference & $J(\mathrm{~Hz})$ \\
\hline 1 & 3.8515 & 3 & singlet & $-\mathrm{OCH}_{3}$ & - \\
\hline 2 & 6.5668 & 1 & singlet & Ar-Hb & - \\
\hline 3 & $6.9478-7.3144$ & 6 & multiplet & Ar-Hc-c'”'” & - \\
\hline 4 & 7.5257 & 2 & singlet & Ar-Hdd' & - \\
\hline 5 & 9.7431 & 1 & singlet & Ar-He & - \\
\hline 6 & 12.4439 & 1 & singlet & -NHf & - \\
\hline
\end{tabular}


Figure S5 ${ }^{13} \mathrm{C}$ NMR spectrum of VMDP-1 along with peak integral
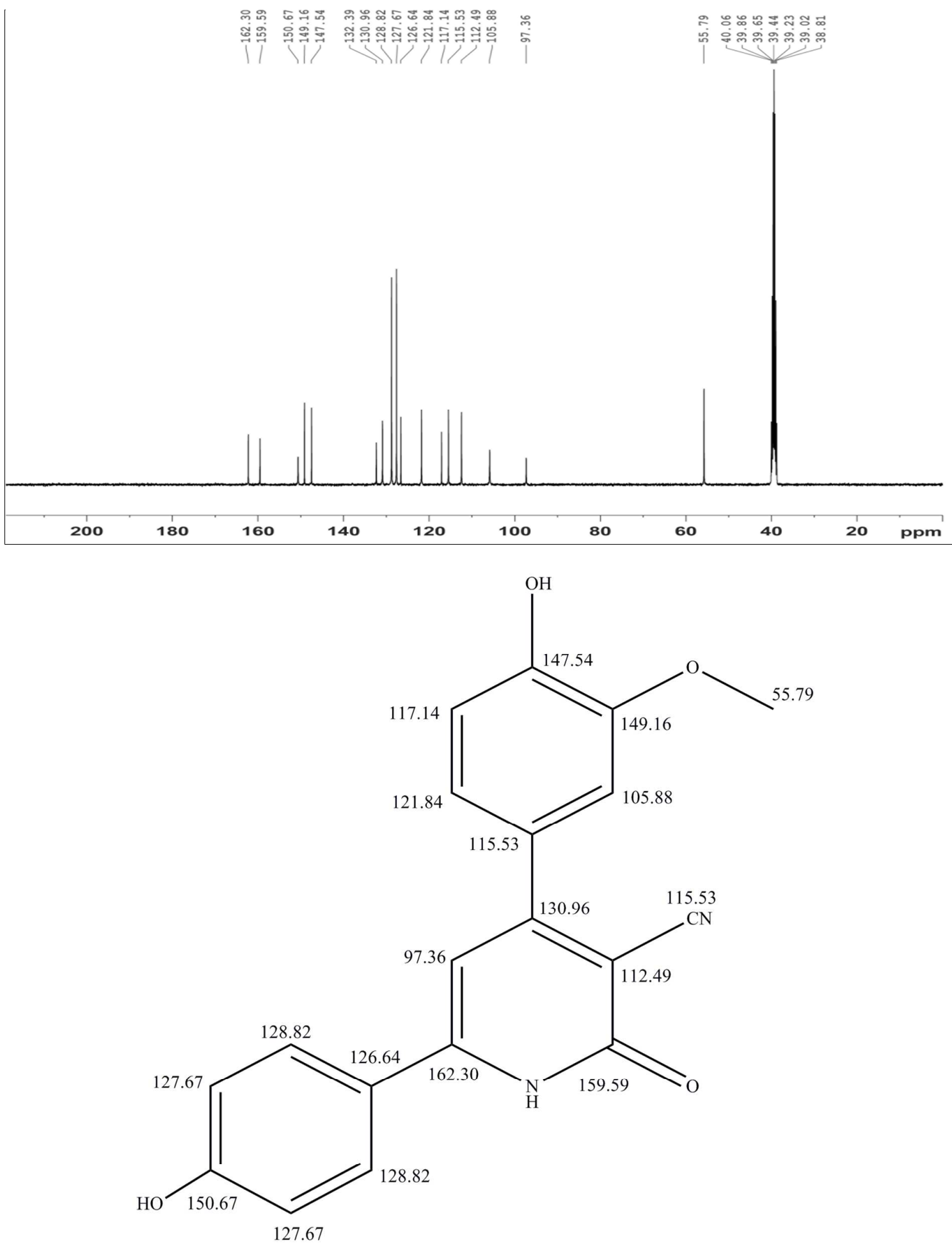
Figure S6 ${ }^{13} \mathrm{C}$ NMR spectrum of VMDP-2 along with peak integral
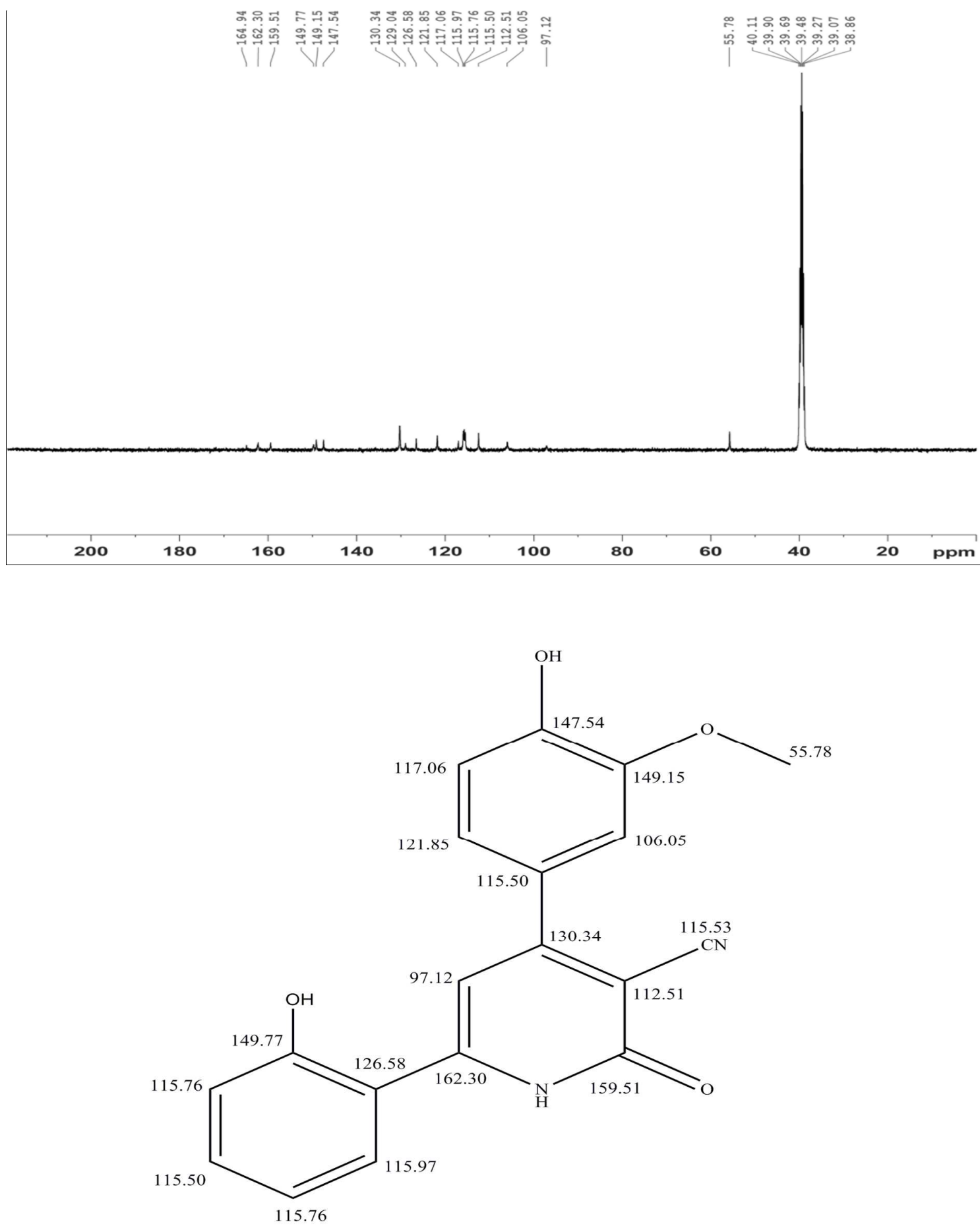
Figure S7 ${ }^{13} \mathrm{C}$ NMR spectrum of VMDP-3 along with peak integral
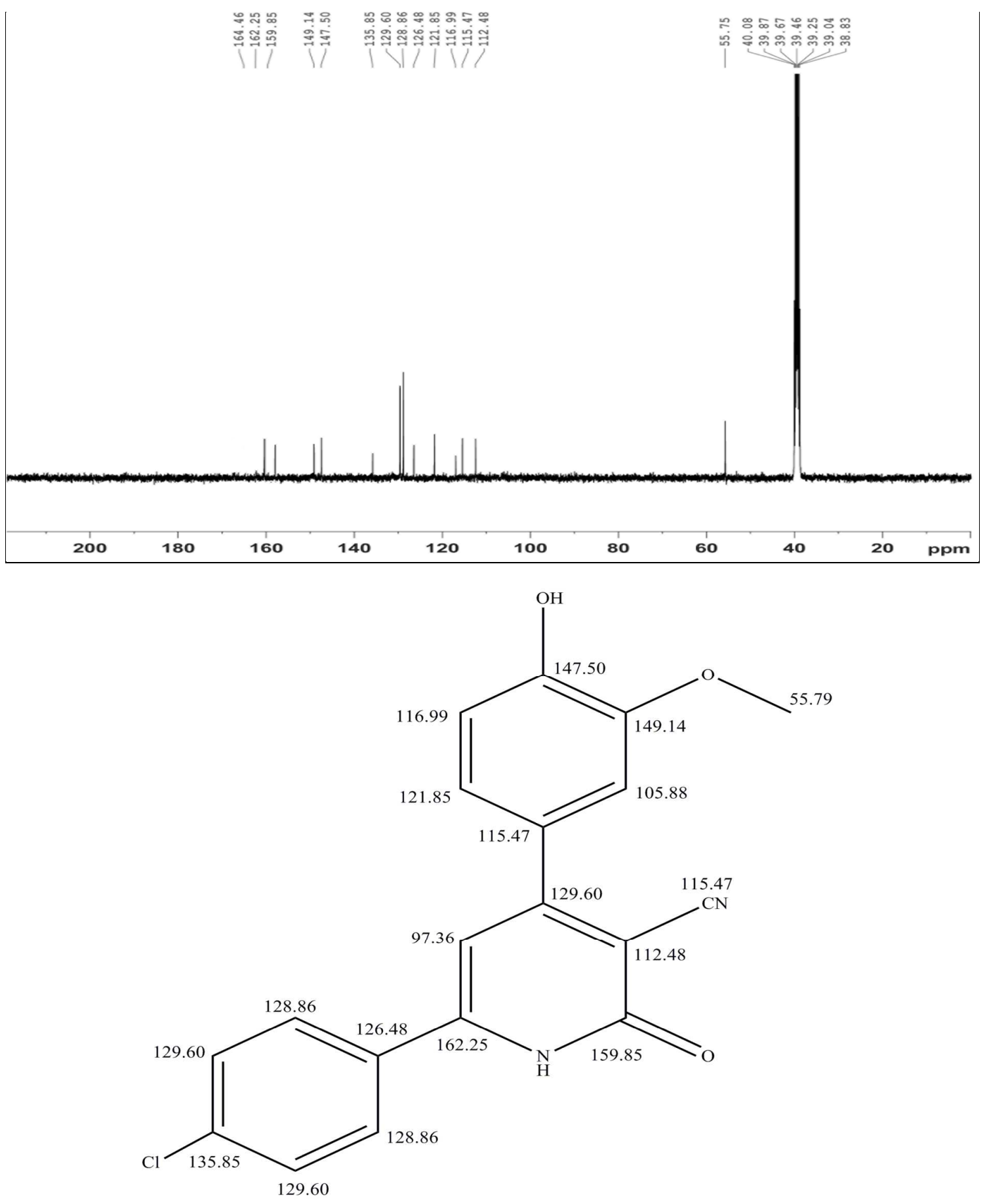
Figure S8 ${ }^{13} \mathrm{C}$ NMR spectrum of VMDP-4 along with peak integral
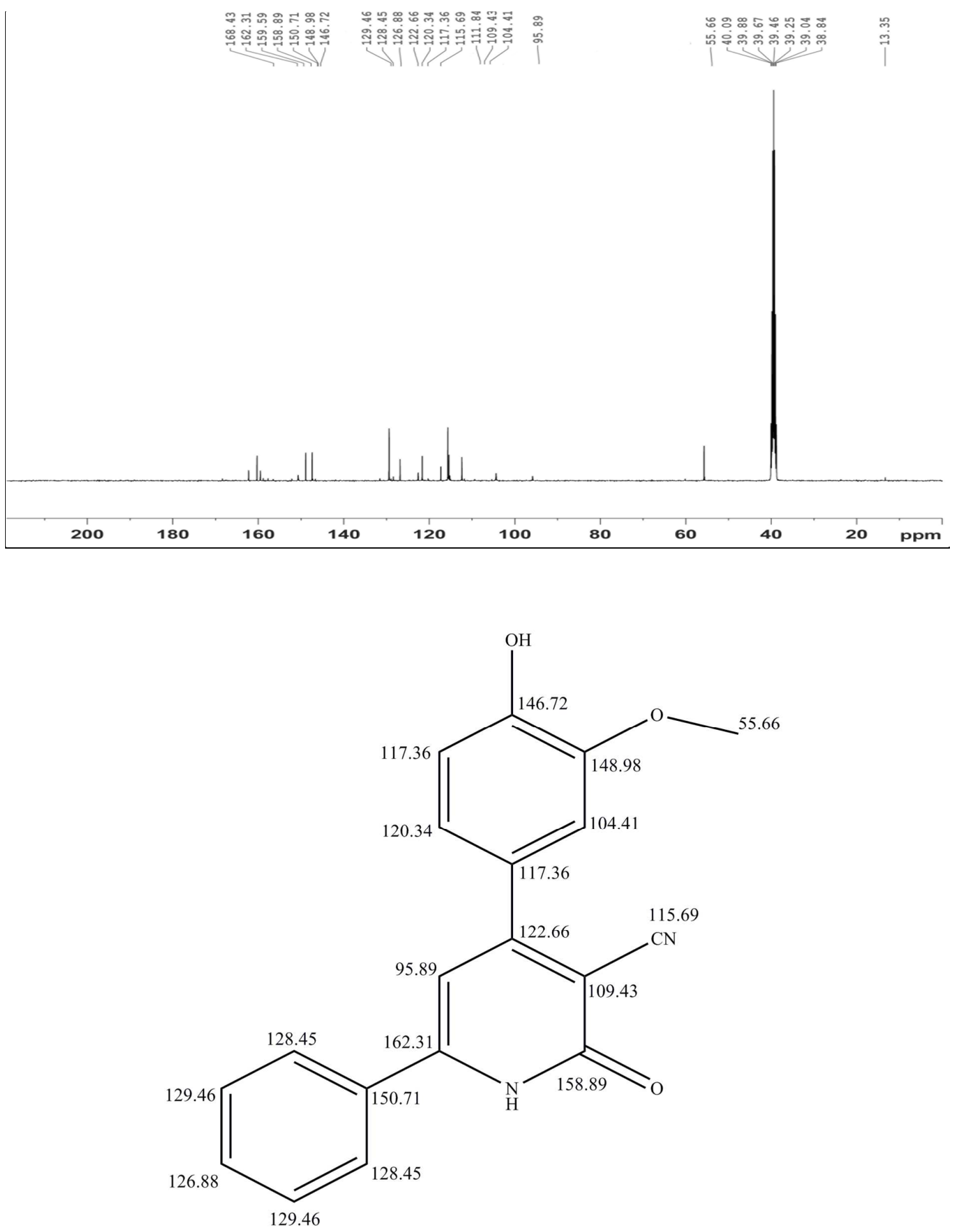
Figure S9 DSC thermogram of standard indium metal

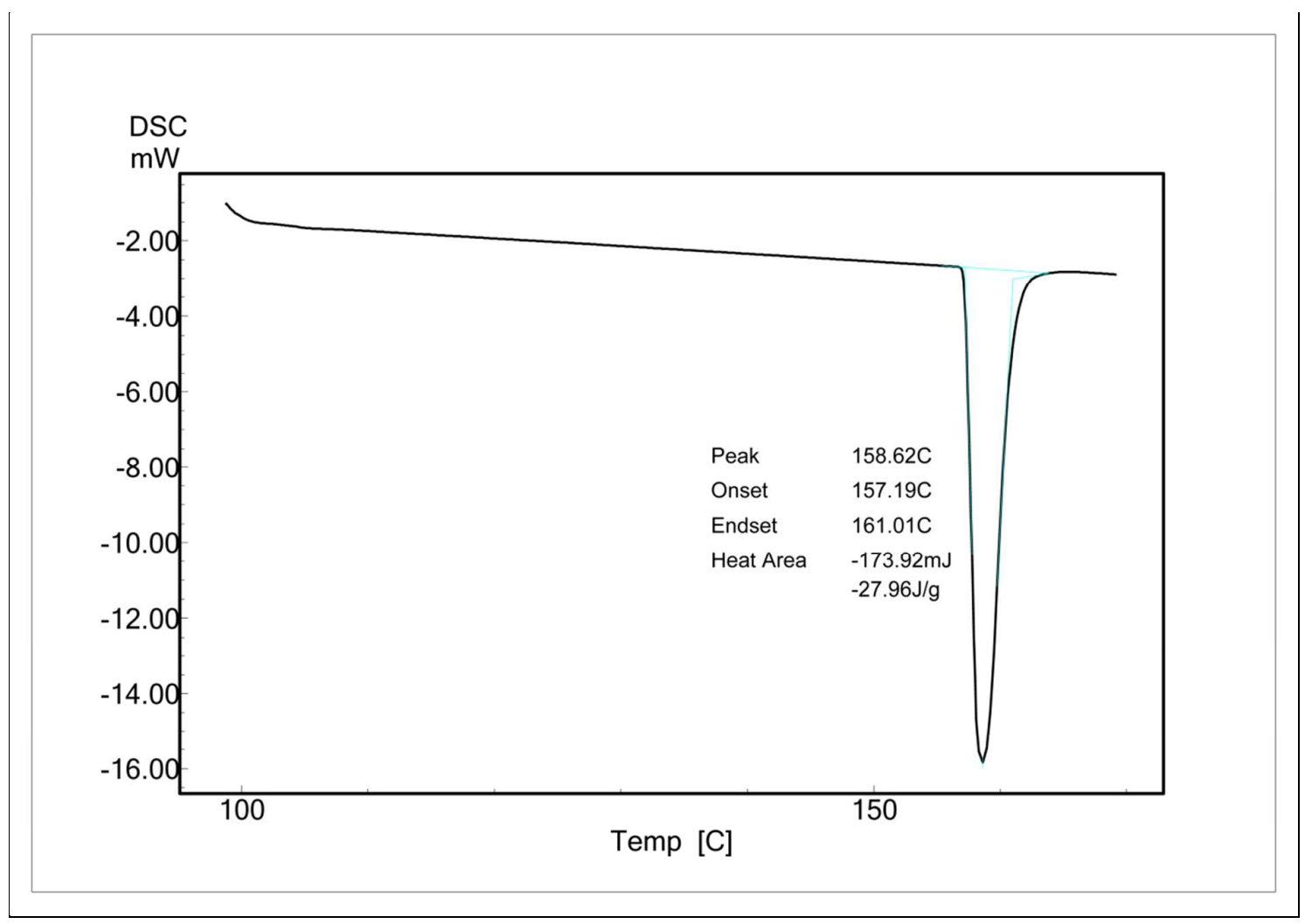


Figure S10 DSC thermogram of standard tin metal




Figure S11 DSC thermogram of VMDP-1

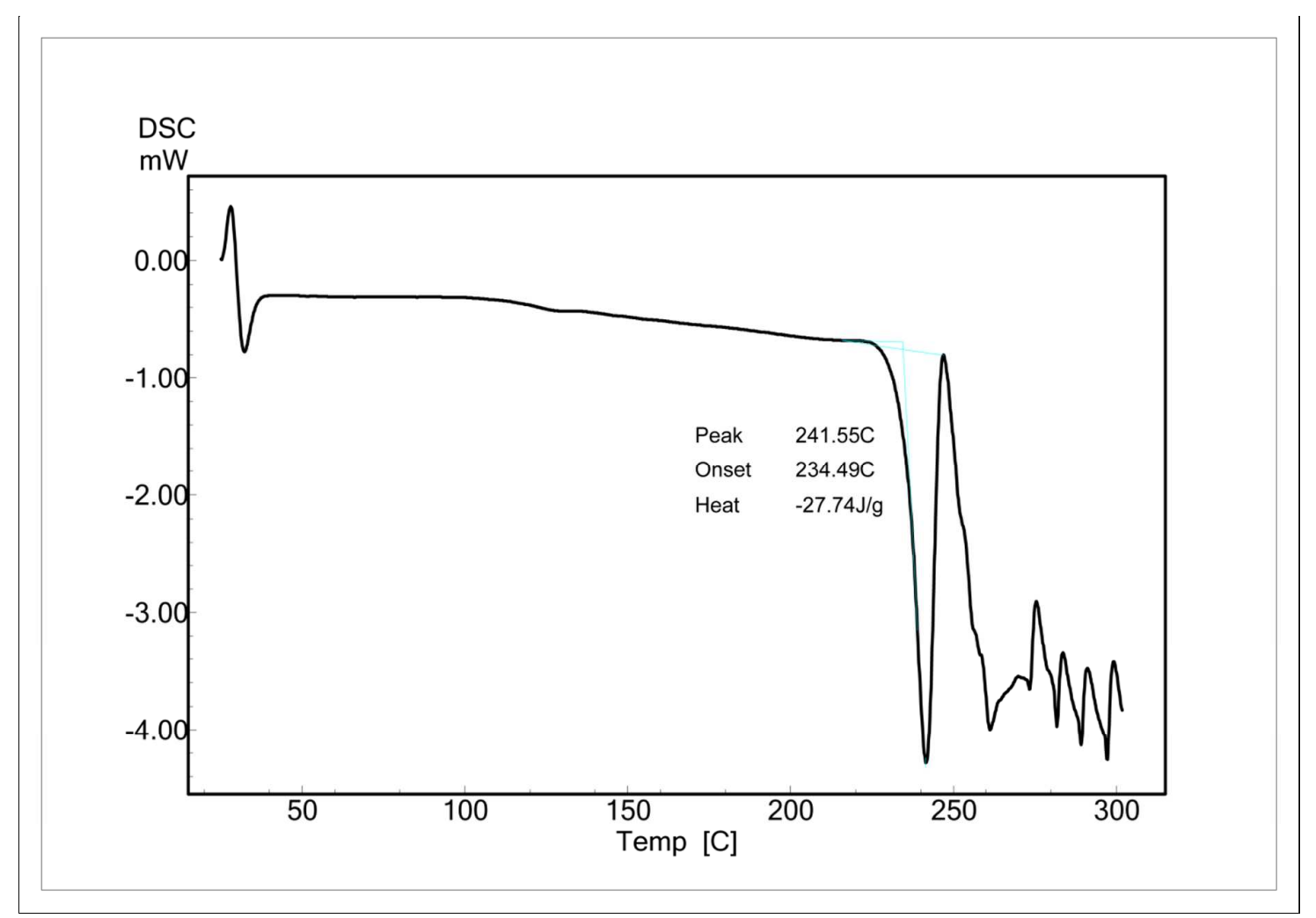


Figure S12 DSC thermogram of VMDP-2

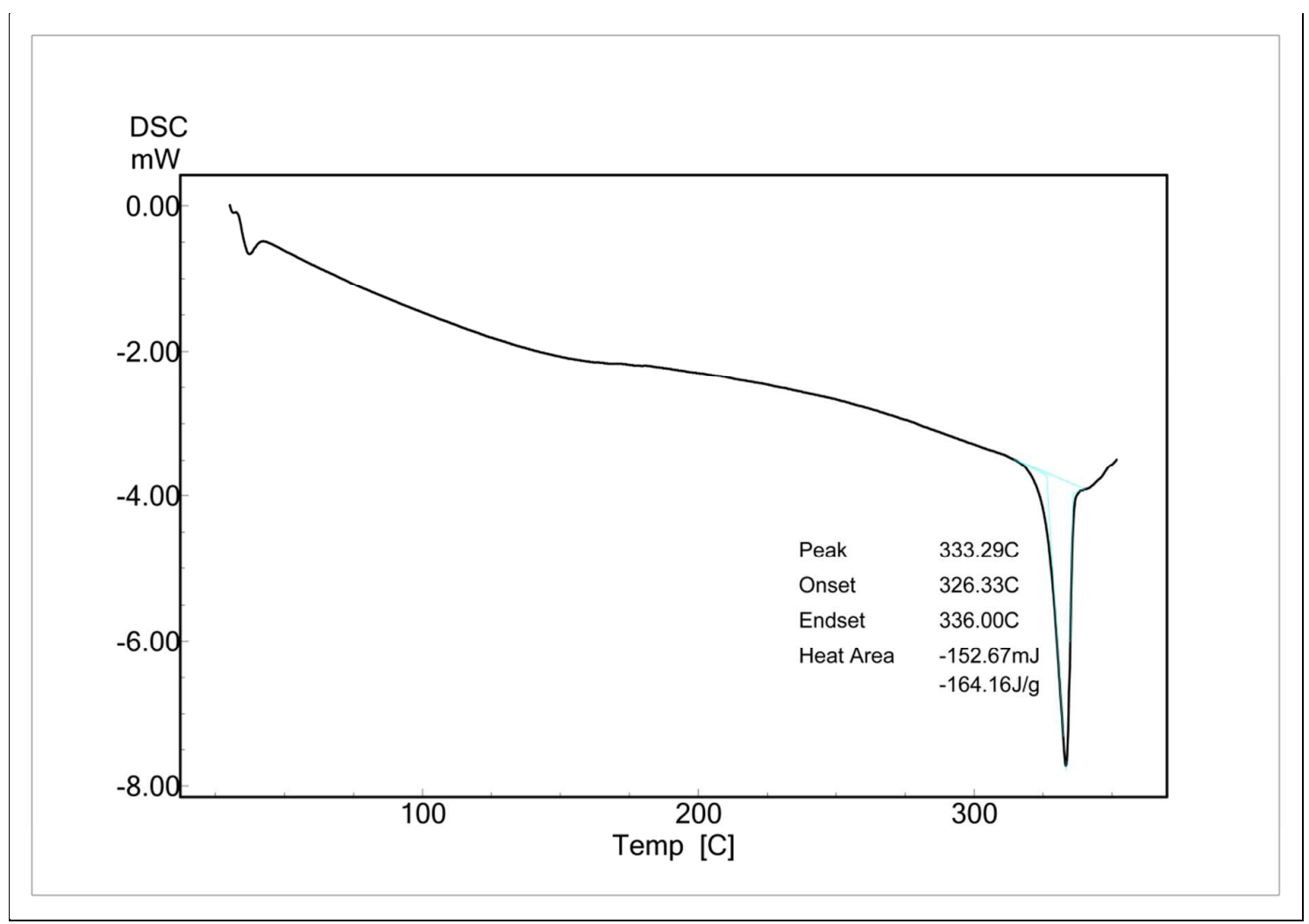


Figure S13 DSC thermogram of VMDP-3

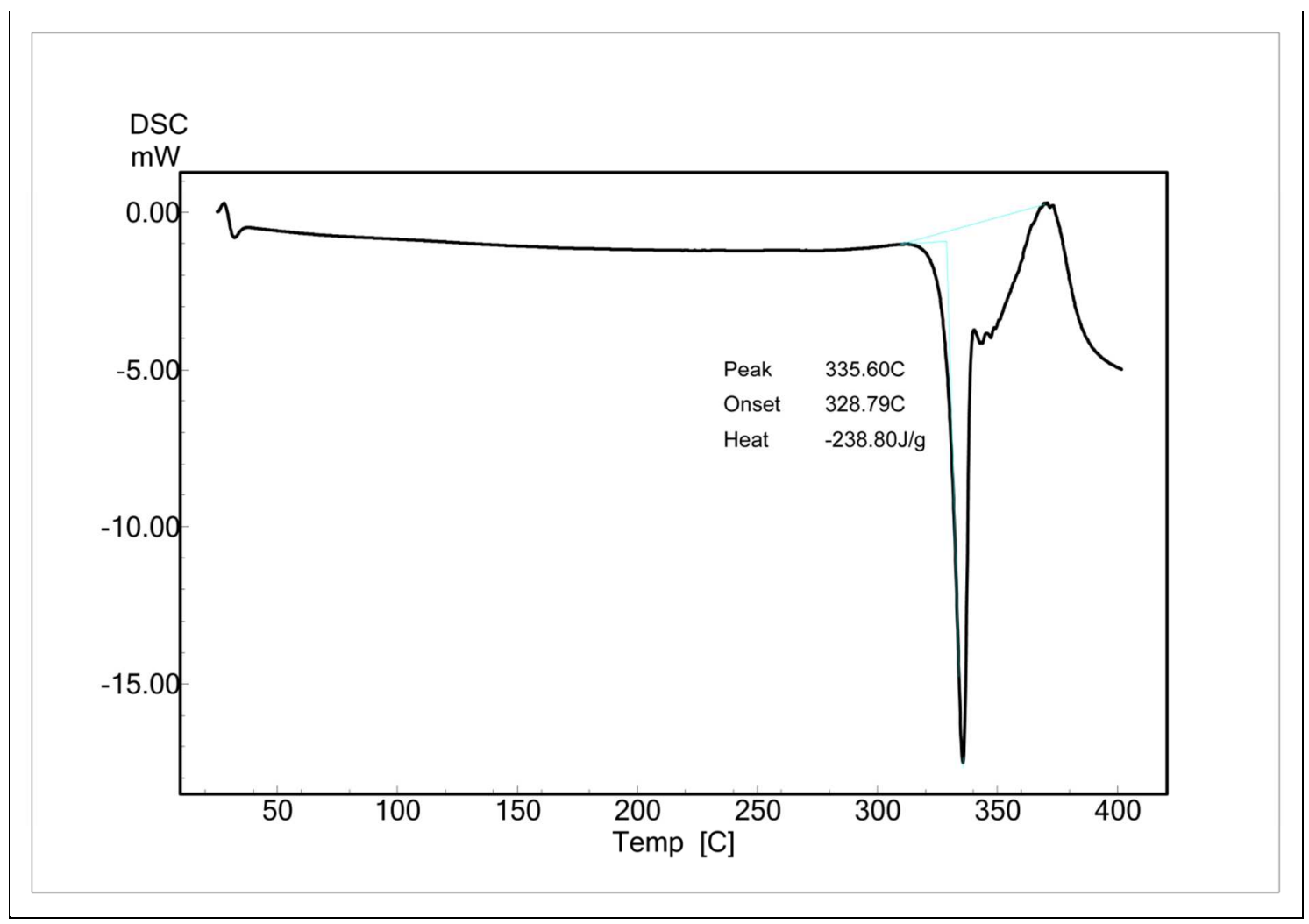


Figure S14 DSC thermogram of VMDP-4

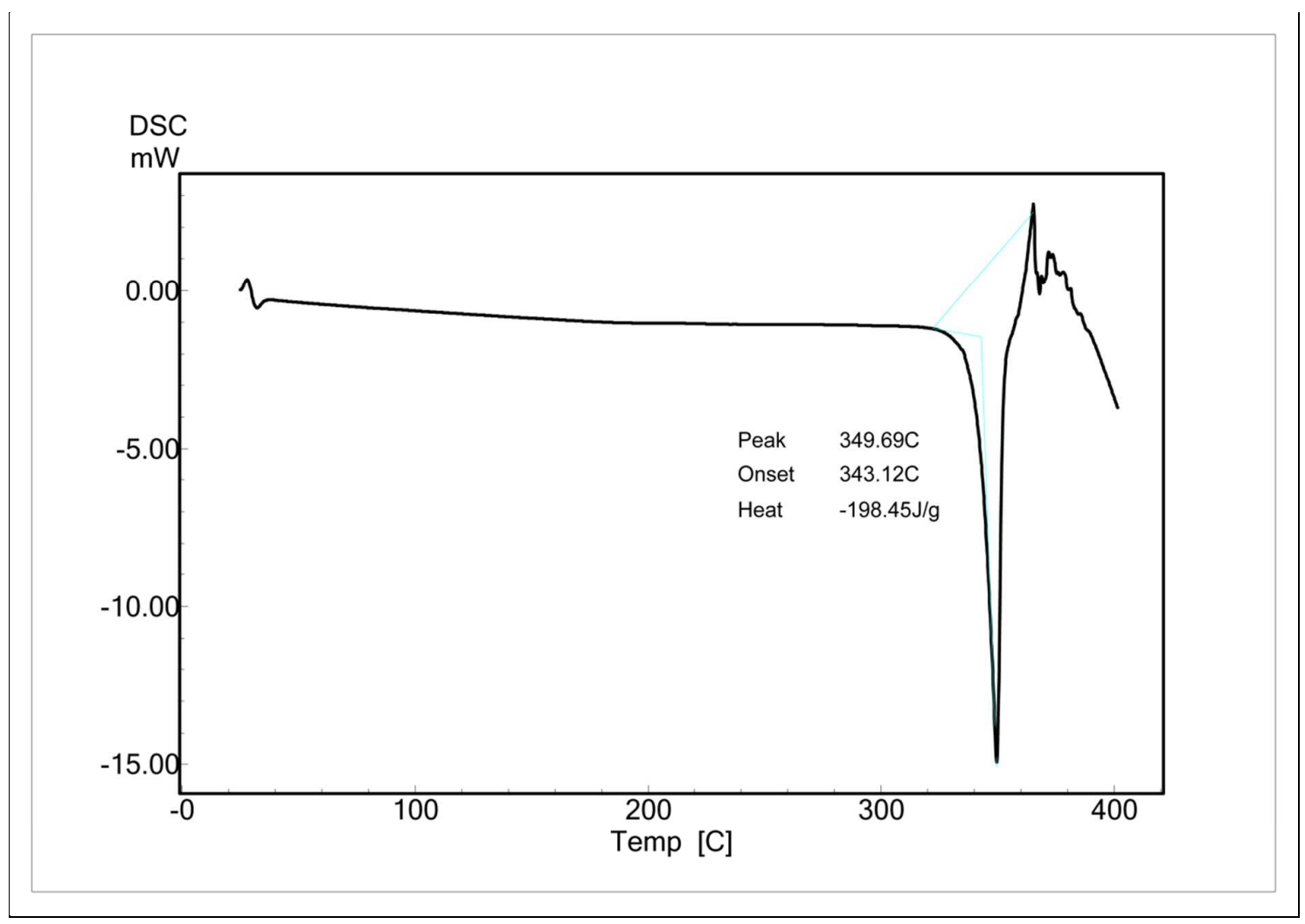

